\title{
Industrielle Dialyseanbieter - zwischen Kommerz und Ethik
}

Unter den berufspolitischen Diskussionen der Nephrologen stach auf dem Kongress der Fachgesellschaft der Nephrologen (DGfN) das Thema „industrielle Dialyseanbieter" heraus. Die Versorgungslandschaft wird durch die zunehmende Anzahl solcher Dienstleister verändert, kritische Stellungnahmen blieben nicht aus. Die DGfN hat dazu Stellung bezogen.

In Deutschland gab es im Mai 2016 nach Angaben der DGfN 1.042 ambulante Dialyseeinrichtungen. In der Hand von kommerziellen Anbietern (z. B. Nephro-Care, B. Braun Avitum, DaVita oder Diaverum) waren zu diesem Zeitpunkt $11 \%$ - mit klar steigender Tendenz. Das hat dazu geführt, dass unverhohlen von einer Industrialisierung der Dialyse gesprochen wird, von einer Etablierung von Dialyseketten.

Tatsächlich haben diese industriellen Dialyseanbieter (Trägerschaft durch kommerziell orientierte Anbieter) nach Ansicht der DGfN die „Dialyselandschaft" bereits merklich verändert, sie sind mittlerweile fester Bestandteil der nephrologischen Versorgung.

Sie können und sollen seitens der Fachgesellschaft daher nicht ignoriert werden. In ihrer Stellungnahme legt die DGfN ihren Umgang mit den industrialisierten Anbietern dar und zeigt Möglichkeiten der Integration, aber auch klare Abgrenzungen auf.

Eine Kernforderung: Dialyseanbieter sollten das gesamte Spektrum der nephrologischen Versorgung anbieten. Dazu heißt es: "Der DGfN liegt es besonders am Herzen, darauf hinzuweisen, dass die nephrologische Versorgung mehr als die Erbringung der Dialyse umfasst. Zu nennen sind hier die Transplantationsnachsorge wie auch die Betreuung von nicht dialysepflichtigen Nierenpatienten, gerade auch im Hinblick auf die Verlangsamung der Krankheitsprogression und Abwendung bzw. dem Hinauszögern der Dialysepflichtigkeit." Die ausschließliche Spezialisierung auf die Dialyse ist demnach nicht im Sinne der Gesellschaft. Vielmehr müsse ein Schwerpunkt in der Prävention liegen, die darauf zielt, dass möglichst viele Patienten möglichst lange dialysefrei leben können.

\section{Seniorenprogramm von Eurotransplant: Alte Nieren für alte Spender}

Patienten mit terminaler Niereninsuffizienz im Alter von 65 Jahren und älter müssen meistens über fünf Jahre warten, bis eine Spenderniere zugeteilt wird. Etwa die Hälfte von ihnen erlebt diesen Tag nicht mehr. Ein Ausweg aus diesem Dilemma wird im Seniorenprogramm von Eurotransplant gesehen, wie Prof. Johan W. de Fijter von der Universität Leiden in seinem Plenarvortrag über "Marginale Spender" ausführte.

Ü ber $50 \%$ der neuen Patienten im Nierenersatztherapie-Programm sind 65 Jahre alt und älter. Nur 5 bis $10 \%$ von ihnen werden eventuell für eine Transplantation gelistet. Aber etwa die Hälfte davon hätte nach geltenden Leitlinien keine Kontraindikation für eine Transplantation.

In der Fachwelt gibt es kaum Zweifel, dass die Nierentransplantation auch im Alter noch sicher und erfolgreich sein kann. Im Vergleich zur Dialyse wird die Überlebenszeit sogar verdoppelt. Selbst bei - jedoch selektierten - über 70-jährigen Organempfängern trifft dies zu.

Tatsache ist aber, dass die Seniorinnen und Senioren eine kürzere Lebenserwartung haben können als das transplantierte Organ. Daher gewinnt die Aktion „Alt für Alt" immer mehr an Bedeutung, denn auch die Nierenempfänger von älteren Spendern leben länger und besser als in der Dialysetherapie.
Eurotransplant hat deshalb ein „Senioren-Programm“ (Eurotransplant Senior DR-compatible Programm, ESP) aufgelegt.

Die Risiken dürfen den Patienten nicht verschwiegen werden. Der Erfolg einer Transplantation von „marginalen Organen" hängt wesentlich vom Gesundheitszustand des Empfängers ab, genauer: vom biologischen Alter des $\mathrm{Pa}$ tienten. In die Waagschale fällt auch die Gesamtzeit, die er schon an der Dialyse verbracht hat und sein Immunstatus nach der Transplantation, denn opportunistische Infektionen post transplantationem sind eine häufige Todesursache.

Zusammenfassend schlug Prof. Johan W. de Fijter von der Universität Leiden folgendes Zuteilungs-Prozedere von marginalen Organen vor:

-Alters-Matching bei über 65-Jährigen. Es wird eine bessere Organüberlebensdauer erreicht, wenn eine alte

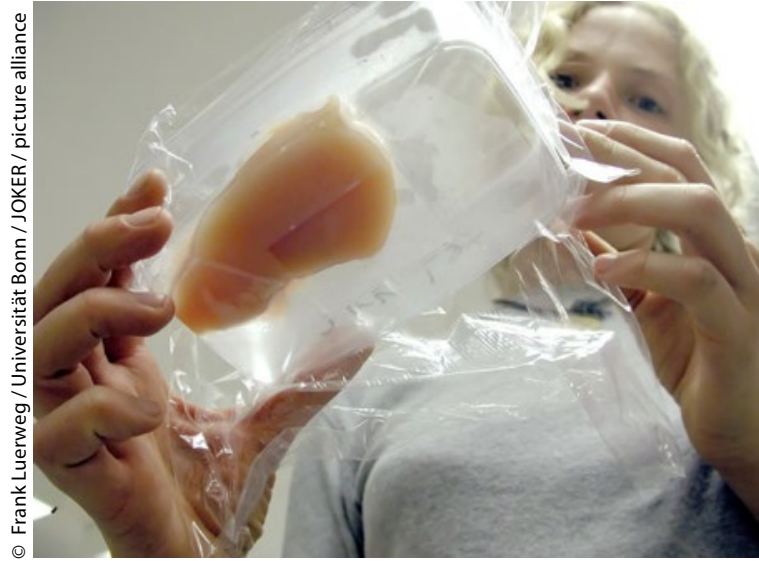

Der Erfolg einer Transplantation ist gröBer, wenn eine alte Spenderniere einem alten Menschen implantiert wird.

Niere einem alten Patienten eingepflanzt wird. Allerdings weiß man auch, dass die Abstoßungsrate bei älteren Spenderorganen höher ist.

-HLA-DR-Matching. Wichtig, um akute Abstoßungsreaktionen und seine Therapie zu verhindern und die Häufigkeit von Infektionen zu reduzieren. - Regionale-lokale Allokation bevorzugen. Möglich ist dies bei „alten“ Nieren von "kontrollierten“ Spendern nach Herzstillstand (DCD). Dadurch kann auch die kalte Ischämiezeit möglichst kurz gehalten werden $(<18$ bis 20 Stunden).

Dr. med. Jochen Aumiller 